\title{
Gamma-Ray Emission from X-Ray Binaries
}

\author{
C.R. Shrader \\ Astrophysics Science Division, Code 661, NASA Goddard Space Flight Center \\ Greenbelt, $M D 20771$
}

\begin{abstract}
We summarize the current observational picture regarding high-energy emission from Galactic X-ray binaries, reviewing the results of the Compton Gamma Ray Observatory mission. We speculate on the prospects for the GLAST era.
\end{abstract}

Keywords: X-ray binaries - gamma rays - neutron stars - jets.

PACS: $97.80 . J p$

\section{INTRODUCTION}

There were some intriguing hints from the CGRO mission, and the prospects have been significantly bolstered in recent years with the firm establishment of $\mathrm{TeV}$ emission from at least $3 \mathrm{X}$-ray binaries. An additional development has been the discovery by the INTEGRAL satellite that there may be a substantial population of highly absorbed, HMXBS and in particular sg XRBs. Possible links between the INTEGRAL sources, the so called micro-quasar XRB subclass and the extragalactic ULX population have been noted. Although the term "micro-Quasar" is in frequent use in the literature, two classes of models - one involving Comptonization or particle-particle interactions associated with relativistic jets, and the other involving wind-wind interactions between the early-type primary and an embedded (young) pulsar are currently under serious discussion. Although one object, PSR 1259-63, is clearly of the latter category, the other two cannot be unambiguously delineated with current observational data. GLAST is expected to provide new insights, which we will put into context of the current observational picture.

\section{THE CGRO ERA}

During the 9-year CGRO mission, EGRET covered the whole sky to varying flux limits, typically $-5 \times 10^{-7} \mathrm{~cm}^{-2} / \mathrm{s}$. No emission was unambiguously associated with Galactic X-ray Binaries. The most compelling case was LSI +61 303, but orbital modulation not identified. Some additional cases of interest: ToO outbursts of 8 prominent X-ray novae, 3 Be transients, as well as large cumulative exposure of Cyg X-1, GX339-4, etc. Suggestive associations or marginal measurements enumerated here:

- LSI +61 303 \& LS 5039: Possibly ambiguous spatial associations with 3E catalog error boxes were made, but the associations are now widely believed due to recent $\mathrm{TeV}$ results, including orbital flux modulation which is now seen, as are apparent $\gamma \gamma$ absorption effects in LS5039. LSI +61303 has a Be star primary (as does another TeV XRB PSR 1259-63), and LS 5039, which also exhibits orbitally-modulated TeV emission has an O7 V [1,2,3].

- There were reports of an unidentified 3E catalog source association with SAX J0635+0533[4], but the significance of the association was marginal [4].

- Marginal evidence for Cen X-3, based on a folding analysis (at the nominal 4.8-s spin period, updated with a concurrent BATSE-generated ephemeris) using the concurrent BATSE frequency, with about a $4 \sigma$ detection [5].

- GRO J1655-40, discovered in 1994, is still the brightest radio source among this class peaking at $\sim 7 \mathrm{Jy}$. An EGRET UL based on prompt ToO observations was reported by [6]. The EGRET observations were not quite simultaneous with the radio peak, and the jet is inclined to the line of sight by perhaps $\sim 70$ degrees.

- GRO J0422+32 was discovered in 1992, and rose to a peak of $\sim 3$-Crab, remaining in the LHS throughout. It was an unresolved, $\sim \mathrm{mJy}$ radio source [7]. Deep EGRET coverage was obtained, but there was no detection. 
- It is notable that the prototypical Be system, A0535+26, was a Z-axis ToO in 1994 when it underwent a $\sim 4-C r a b$ peak flux outburst. No high energy $\gamma$-rays were detected. A0535+26 is in the heavily exposed (and highly structured) Taurus region which contains an EGRET source candidate, but with a large error box. Another major Be event was GRO J1008-57, which was also an EGRET non-detection.

- Cyg X-3 was also a Z-axis ToO in 1991 during an transient radio outburst, and the Cygnus region was heavily exposed. Again, no high-energy $\gamma$ 's were detected.

SS 433, which is the only known case of a hadronic jet, was observed extensively and not detected; UL $\sim 5 \times 10^{-7}$

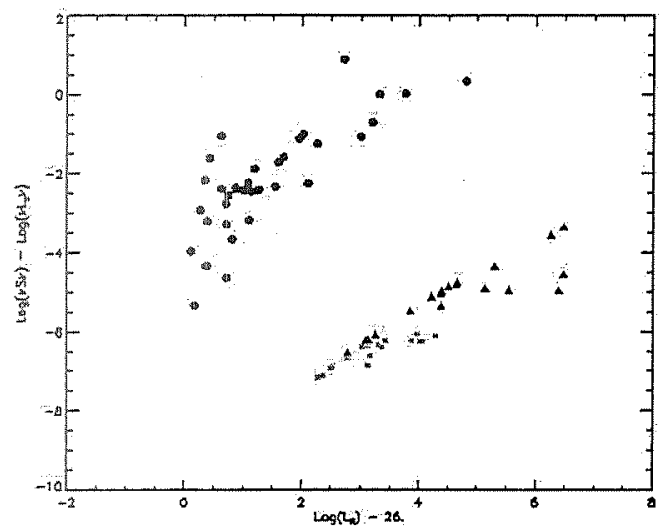

Figure 1. The relative power of the spectral components which may be associated with a disk or that scale with the bolometric luminosity - X-rays in Galactic binaries and optical in radio loud QSOs - poses a distinct contrast: The radio-loud QSO's have $\sim 2$ order of mag higher "disk-to-jet" power than XRBs. Also note the delineation between neutron star systems ("), and black-hole systems ( $\mathbf{A}$ ) among the galactic sources. This is the case despite the fact that the distribution of bulk-Lorentz factors for X-ray binary radio jets is approximately consistent with that of quasars.

\section{Micro-Quasars: A Misnomer?}

The term micro-quasar is loosely applied loosely in the literature and is lacking a rigorous definition. Typically, accompanying cartoon diagrams consist of a disk accretor with collimated outflow, characterized by resolved radio structure. The "canonical" examples, e.g. GRO J1655-40, GRS 1915+105, are further characterized by sporadic high-amplitude variability or episodic behavior. The known $\gamma$-ray loud XRBs on the other hand are low-luminosity $\mathrm{X}$-ray sources, consistent with wind accretion rather than roche-lobe overflow. The flat X-ray light curves of LSI +61303 and LS 5039 are entirely distinct from those of the prototypical objects. PSR 1259-63 is definitely different (48-ms pulsar in Be system), and recent evidence based on VLBI imaging on $\sim$ mas scales suggests that the radio emission from LSI +61303 exhibits a parabolic morphology consistent with wind-wind emission scenarios $[8,9]$.

The key issues that GLAST should be able to resolve are (i) whether or not any of the marginal EGRET candidates are to be believed (ii) does the gamma-ray emission from LSI +61 3030 and/or LS 5039 vary in a way with other properties of those systems (such as the radio or X-ray light curves or Ha EW history), (iii) most significantly is gamma-ray emission a characteristic of a broader subclass of X-ray binaries which are unambiguously accretion powered,.

\section{REFERENCES}

1. Tavani, M., et al., 1998, ApJ, 497, L89

2. Aharonian, F., et al., 2006, A\&A, 460, 743

3. Albert, J., 2006, Sci., 312, 1771

4. Kaaret, P., et al, 1999, ApJ, 523, 197

5. Vestrand, T., 1997, ApJ, 483, L49.

6. Levenson, A., \& Mattox, J.R., 1996, 462, L67

7. Shrader, C.R., et al., 1994, ApJ, 434, 698

8. Dermer, C.D., Boettcher, M., 2006, ApJ, 643, 1081

9. Dubus, G., 2006, astro-ph/0608262 